\title{
Uso de psicotrópicos por pessoas idosas com hipertensão: prevalência e fatores associados
}

\author{
Use of psychotropic drugs by older adults with hypertension: prevalence and associated factors
}

\author{
Paula Antunes Bezerra Nacamural (D) \\ Luana Cristina Bellini Cardoso' (D) \\ Anderson da Silva Rêgo ${ }^{1,2}$ (D) \\ Rafaely de Cássia Nogueira Sanches' ${ }^{1}$ \\ Cremilde Aparecida Trindade Radovanovic ${ }^{10}$ \\ Marcelle Paiano' (ID
}

\section{Resumo}

Objetivo: analisar os fatores associados ao uso de psicotrópicos por pessoas idosas com hipertensão arterial (HA) acompanhadas pela atenção primária. Método: estudo transversal, realizado em um município do noroeste do estado do Paraná, Brasil. A coleta de dados foi realizada com pessoas idosas, no primeiro semestre de 2016, por intermédio de um instrumento adaptado e validado para avaliação da satisfação com os serviços da Atenção Primária à Saúde. Utilizou-se o modelo de regressão logística, com o método stepwise e a magnitude das associações foi estimada pelo cálculo de Razão de Prevalência (RP). Resultados: participaram do estudo 260 pessoas idosas com HA, das quais 25,4\% faziam uso de psicotrópicos, sendo a maioria com idade entre 60 e 69 anos $(48,8 \%)$ e do sexo feminino (67,3\%). A análise multivariada evidenciou que o uso de psicotrópicos é mais prevalente em idosos que fazem uso de tabaco (RP: 4,09; IC95\%: 1,81-9,18), com circunferência abdominal alterada (RP: 2,58; IC95\%: 1,29-5,18), com obesidade (RP: 2,43; IC95\%: 1,30-4,55) e aos que relataram ter efeitos colaterais dos medicamentos utilizados no tratamento da HA (RP: 2,98; IC95\%: 1,23-7,21). No contexto organizacional e relacionais das equipes da estratégia saúde da família, em idosos com hipertensão e que fazem uso de psicotrópicos observou-se maior prevalência na insatisfação com o atendimento (RP: 6,71; IC95\%: 1,37-32,71) e com a falta de apoio e de compreensão com os problemas relatados durante as consultas (RP: 2,17; IC95\%: 1,11-4,25). Conclusões: Por ser um problema de saúde pública e que atinge parcela significativa da população com maior risco de agravos à saúde, destaca-se a importância de pesquisas nessa área, as quais busquem alternativas para melhorar a qualidade de vida da pessoa idosa com comorbidades e que utilizam psicotrópicos por meio de um cuidado integral.

\footnotetext{
Universidade Estadual de Maringá, Departamento de Enfermagem, Programa de Pós-graduação em Enfermagem. Maringá, Paraná, Brasil.

2 Centro Universitário Ingá (UNINGÁ), Departamento de Enfermagem, Programa de Pós-graduação em Enfermagem. Maringá, Paraná, Brasil.
}

Financiamento: Coordenação de Aperfeiçoamento de Pessoal de Nível Superior (CAPES), Processo no 88882.449287/2019-01.

Os autores declaram não haver conflito na concepção deste trabalho.
Palavras-chave: Idoso. Hipertensão. Psicotrópicos. Prescrições de Medicamentos. Tratamento Farmacológico. Atenção Primária à Saúde. 


\section{Abstract}

Objective: to analyze the factors associated with the use of psychotropic drugs by older adults with hypertension treated in primary care. Method: a cross-sectional study was carried out in a city in the northwest of the state of Paraná, Brazil. Data collection was carried out with older adults in the first semester of 2016, using an adapted validated instrument for assessing satisfaction with Primary Health Care services. The logistic regression model was used with the stepwise method and the magnitude of the associations was estimated by calculating Prevalence Ratio (PR). Results: 260 older adults with hypertension participated in the study, of which $25.4 \%$ were in use of psychotropic drugs, most were aged 60-69 years $(48.8 \%)$ and female $(67.3 \%)$. The multivariate analysis showed psychotropic drugs use was more prevalent in participants who used tobacco (PR: 4.09; 95\%CI: 1.81-9.18), had abnormal waist circumference (PR: 2.58; 95\%CI \%: 1.29-5.18), were obese (PR: 2.43; 95\%CI: 1.30-4.55) and reported side-effects of drugs used in AH treatment (PR: 2, 98; 95\%CI: 1.23-7.21). Regarding the organizational and relational aspects of the family health strategy teams, participants with hypertension and in use of psychotropic drugs had a higher rate of dissatisfaction with the service (PR: 6.71; 95\%CI: 1.37-32, 71) and with lack of support and understanding of the problems reported during consultations (PR: 2.17; 95\%CI: 1.11-4.25). Conclusions: As a public health problem that affects a significant contingent of the elderly population at high risk of health problems, further studies should be conducted in this area. Future studies should seek alternatives to improve the quality of life of elderly with comorbidities and in use of psychotropic drugs through comprehensive care.

\section{INTRODUÇÃO}

O uso excessivo de medicamentos faz parte da cultura ocidental, cuja convicção de que o malestar, bem como o sofrimento de todo gênero deve ser sanado a qualquer custo. Diante desses problemas, a prática do uso da polifarmácia tem se tornado um dos caminhos mais eficientes e rápidos para minimizar o sofrimento psíquico ${ }^{1}$. Os medicamentos psicotrópicos (psique $=$ mente $\mathrm{e}$ tropico = alteração), de acordo com a Organização Mundial da Saúde (OMS) são modificadores seletivos do Sistema Nervoso Central (SNC), ocasionando alterações no comportamento, humor e cognição. O uso desses medicamentos é indispensável na terapêutica de alguns transtornos mentais ou distúrbios psiquiátricos, tais como: ansiedade, insônia, depressão, agitação, convulsão e psicose ${ }^{2}$.

Desta forma, a utilização de psicotrópicos temse elevado consideravelmente, especialmente os antidepressivos, devido a melhora nos diagnósticos de transtornos mentais, o aparecimento de novos fármacos no mercado e as novas indicações terapêuticas já existentes ${ }^{3}$. Especificamente para os antidepressivos, além desses fatores, deve- se considerar o prolongamento do tratamento medicamentoso da depressão; no caso das pessoas idosas, também da depressão na fase final de vida, em função das limitações físicas e sociais da idade . $^{4}$.

Entre os psicotrópicos comumente encontrados em uso contínuo a outras classes, enquadram-se medicamentos como: antipsicóticos, barbitúricos, benzodiazepínicos, neurolépticos e antidepressivos, os quais podem desencadear eventos adversos severos, que vão desde o aumento dos riscos de acidente vascular cerebral, aumento da mortalidade, desenvolvimento de dependência física e da tolerância na indução do sono, até o aumento do comprometimento cognitivo, risco de queda e convulsões ${ }^{3,4}$.

As pessoas idosas estão entre aquelas com maior probabilidade de receber a receita de um benzodiazepínico, tornando-se uma importante preocupação de saúde pública ${ }^{5}$. Nesse sentido, um estudo de base populacional, realizado no município de Campinas, no estado de São Paulo, constatou prevalência de $10,8 \%$ do uso de psicotrópicos entre os idosos ${ }^{6}$. Tal número pode diferir caso o indivíduo esteja na comunidade ou institucionalizado. Pesquisas 
com idosos em instituições de longa permanência detectaram que entre $59,7 \%$ e $74,6 \%$ utilizavam essas medicações, sendo que o maior consumo foi em países da Europa e com predomínio de antipsicóticos ${ }^{7,8}$. Entre as pessoas idosas residentes na comunidade a prevalência variou de $9,3 \%$ a 37,6\%, com predominância dos benzodiazepínicos ${ }^{8}$. $\mathrm{Na}$ população idosa brasileira, observou-se que $21,7 \%$ consumiam benzodiazepínicos há mais de um ano9

Ao considerar que a incidência de polifarmácia é um fator adjacente a expectativa de vida, o seu acompanhamento torna-se uma prática necessária das equipes da Estratégia Saúde da Família (ESF). A atual política de saúde mental brasileira apresenta a Atenção Primária a Saúde (APS) como porta de entrada e ordenadora do cuidado dos usuários em sofrimento psíquico, e a atuação das equipes suscitam a necessidade em elaborar estratégias incisivas à população idosa, de acordo com essas políticas ${ }^{3,4}$.

Nesse sentido, estudos sobre a utilização de psicofármacos e polifarmácia constituem um campo de investigação inerente a farmacoepidemiologia, e são essenciais para a promoção do uso racional de medicamentos. Além disso, conhecer os fatores associados ao consumo de psicotrópicos por pessoas idosas com hipertensão arterial (HA) acompanhados pela APS pode favorecer a elaboração de estratégias de prevenção e promoção da qualidade de vida da pessoa idosa, evitar a dependência de substâncias e o surgimento de efeitos adversos com o alto consumo dessa classe de medicamentos ${ }^{3-9}$. Diante do exposto, o objetivo deste estudo foi analisar os fatores associados ao uso de psicotrópicos por pessoas idosas com hipertensão arterial acompanhadas pela atenção primária à saúde.

\section{MÉTODO}

Trata-se de uma pesquisa transversal, realizada com pessoas idosas em tratamento da HA e acompanhadas pela APS. O município em que a pesquisa foi realizada localiza-se no noroeste do estado do Paraná, Brasil, que na época da coleta de dados, possuía população estimada de 403.063 habitantes. A APS encontrava-se organizada de forma descentralizada, composta por 34 Unidades Básicas de Saúde (UBS) e 74 equipes da ESF, perfazendo a cobertura de $68,01 \%$ da população ${ }^{10}$.

Considerou-se como critérios de inclusão, pessoas com HA, idade igual e/ou superior a 60 anos, residentes na área urbana do município, possuir prontuário atualizado e ter recebido atendimento por profissional de saúde da UBS nos últimos 12 meses que antecederam a coleta de dados. $\mathrm{O}$ critério de exclusão estabelecido foi não apresentar informações sobre as consultas clínicas e prescrições medicamentosas registradas nos prontuários clínicos.

Para a realização do cálculo amostral, considerou-se o número de pessoas cadastradas no sistema SISHIPERDIA (programa de cadastro e acompanhamento de pessoas com hipertensão e Diabetes Mellitus) até o ano de 2014, que totalizou em 27.741 indivíduos, dos quais, 62,4\% eram idosos. Utilizou-se tal período como base para o cálculo, por considerar que os dados referentes ao ano anterior a coleta de dados (2015), ainda se encontrava em processo de preenchimento, o que poderia comprometer a fidedignidade das informações. Concernente, para determinação do tamanho mínimo da amostra, utilizou-se o cálculo da proporção da população, considerando o erro de estimativa de $5 \%$, intervalo de confiança de $90 \%$. Tendo em vista que o tamanho da população era finito e conhecido, a amostra mínima, de caráter representativo, foi de 252 idosos, posteriormente aleatorizados e estratificados de acordo com o número de pessoas acompanhadas por cada UBS do município, resultando na amostra final de 260 idosos.

Realizou-se o contato inicial com os gestores, médicos e enfermeiros das UBS e posteriormente com as pessoas idosas, sendo apresentado o estudo e realizado o convite para participação. A coleta de dados foi realizada no primeiro semestre de 2016 por três enfermeiros (as), discentes de um programa de pós-graduação de uma universidade pública localizada no município. Para essa etapa, foi realizado treinamento prévio sobre os objetivos do estudo, técnicas de entrevistas e de mensuração dos parâmetros antropométricos e clínicos dos entrevistados. Todas as entrevistas foram individuais e realizadas em ambientes confortáveis e livre de interferência. 
Dois instrumentos foram utilizados para a coleta dos dados. O primeiro foi adaptado e validado por Paes $^{11}$, norteado pelo instrumento Primary Care Assessment Tool (PCATool) e avaliou os serviços prestados pela APS sob a ótica de pessoas com HA. Para este estudo, foram utilizadas as variáveis do bloco de questões referentes ao perfil sociodemográfico e econômico, em que se estabeleceu a escolaridade de acordo com os anos de estudos completos e a raça/cor foi determinada a partir da autodeclaração dos participantes. Investigou-se ainda as questões relacionadas ao uso de medicamentos (divididos segundo os grupos terapêuticos aos quais pertencem) e as questões relacionadas ao vínculo entre profissional e paciente.

O segundo instrumento obteve dados do perfil socioeconômico dos participantes, conforme indicação da Associação Brasileira de Empresas de Pesquisa (ABEP), categorizados, neste estudo, em classes $\mathrm{AB}, \mathrm{C}$ e $\mathrm{DE}^{12}$. O acompanhamento adequando foi definido quando a pessoa idosa compareceu as consultas de rotina pré-agendadas, por no mínimo três vezes ao ano, considerando o ano anterior a coleta ${ }^{13}$.

Após a realização das entrevistas, foram coletadas informações dos prontuários eletrônicos dos participantes do estudo. Os dados referentes aos medicamentos em uso foram extraídos da aba de receituário no prontuário eletrônico e posteriormente transcritos para o instrumento de coleta de dados. A classificação dos medicamentos foi realizada a partir do seu princípio ativo, identificada com o auxílio do Dicionário de Especialidades Farmacêuticas (DEF), e posteriormente agrupados seguindo as diretrizes do Anatomical Therapeutic Chemical Classification System (ATC) ${ }^{14}$. Desse modo, considerou-se como psicotrópicos os: benzodiazepínicos, antidepressivos, inibidores seletivos de recaptação da serotonina, tricíclicos e outros antidepressivos. Para essa análise excluíram-se os antipsicóticos.
A aferição dos parâmetros antropométricos e clínicos foram realizados durante as entrevistas, com equipamentos devidamente atualizados e calibrados, obedecendo as diretrizes sobre as técnicas e procedimentos para verificação das medidas. Para os valores pressóricos, baseou-se nas condutas descritas pela $7^{a}$ Diretriz Brasileira de Hipertensão Arterial, realizadas pela Sociedade Brasileira de Cardiologia $(\mathrm{SBC})^{15}$ Considerou-se Controle Pressórico Adequado quando a pressão arterial sistólica (PAS) apresentou valor $\leq 140 \mathrm{mmHg}$ e pressão arterial diastólica (PAD) $\leq 90 \mathrm{mmHg}^{15}$.

A coleta dos parâmetros antropométricos obedeceu às normas da associação Brasileira para o Estudo da Obesidade e Síndrome Metabólica $(\mathrm{ABESO})^{16} \mathrm{e}$ das recomendações da Caderneta do Idoso, desenvolvida pelo Ministério da Saúde ${ }^{17}$. Utilizou-se fita inelástica para aferir a circunferência abdominal (CA), o qual foi considerado como alterado valores acima de $\geq 94$ centímetros para a população masculina e $\geq 80$ para a população feminina ${ }^{16}$.

A aferição do peso e da altura foi realizada a partir de uma balança antropométrica com estadiômetro acoplado, devidamente calibrada. A partir desses resultados, realizou-se o cálculo do Índice de Massa Corporal (IMC), em que se estabeleceu como peso normal o resultado do cálculo $<22 \mathrm{~kg} / \mathrm{m}^{2}$, peso adequado os que apresentaram IMC $\geq 22 \mathrm{e} \leq 27 \mathrm{~kg} / \mathrm{m}^{2}$ e excesso de peso os valores superiores a $27 \mathrm{~kg} / \mathrm{m}^{2} 16,17$.

O resultado oriundo do cálculo da razão cintura/ estatura (RCE) foi codificado de acordo com estudo sobre o diagnóstico de excesso de peso em pessoas idosas a partir da RCE, sendo considerado alterado os valores $\geq 0,56^{18}$. O Índice de Conicidade (IC) foi verificado por meio da equação proposta por Pitanga e Lessa (Figura 1), como também os pontos de corte, em que todo valor acima de 1,25 para a população masculina e 1,18 para a população feminina foi determinado como alterado ${ }^{19}$. 
A variável dependente foi a utilização de pelo menos um psicotrópico, verificado na análise dos prontuários dos participantes do estudo. As variáveis independentes explicativas foram divididas em três blocos, quais sejam: perfil sociodemográfico e econômico; perfil clínico, antropométrico e hábitos de vida; e variáveis referentes as questões organizacionais e relacionais dos profissionais de saúde das equipes da APS no atendimento à população alvo deste estudo, categorizadas a partir do índice composto das questões referentes aos blocos citados, resultantes da análise de agrupamento nãohierárquico por repartição.

Todos os questionários foram checados, tabulados e posteriormente analisados por meio do software Statistical Package for Social Sciences (SPSS), versão 20.0. Utilizou-se o modelo de regressão logística, com o método stepwise, considerando todas as variáveis que apresentaram o $p$-valor $<0,20$ na análise univariada e se mantiveram no modelo multivariado apenas as variáveis que resultaram o valor de $p<0,05$. A magnitude das associações foi estimada pelo cálculo de Razão de Prevalência (RP), adotando o intervalo de confiança de $95 \%$ como medida de precisão.

Os preceitos éticos estabelecidos pela Resolução 466/12 do Conselho Nacional de Saúde, foram devidamente respeitados, com a aprovação do estudo pelo Comitê de Ética em Pesquisa da instituição signatária (Parecer no 1.407.687/2016). Todos os participantes leram e assinaram o Termo de Consentimento Livre e Esclarecido.

\section{RESULTADOS}

Foram entrevistadas 260 pessoas com HA, com idade entre 60 e 69 anos (48,8\%), sexo feminino $(67,3 \%)$, e que se autodeclararam da cor branca $(64,6 \%)$, convive com companheiro e filhos $(56,5 \%)$ e com baixa escolaridade (62,7\%). Quanto as questões econômicas, a maioria era aposentada e/ou pensionista $(74,2 \%)$ e pertencia ao extrato $C$ $(40,8 \%)$ no que concerne o poder de compra do chefe familiar (Tabela 1).

Tabela 1. Perfil sociodemográfico e econômico das pessoas idosas com hipertensão acompanhadas pela APS (N=260). Paraná, Brasil, 2016.

\begin{tabular}{lc}
\hline Variáveis & $\mathrm{n}(\%)$ \\
\hline Idade & $127(48,8)$ \\
$60-69$ & $96(36,9)$ \\
$70-79$ & $35(13,5)$ \\
$80-89$ & $2(0,8)$ \\
$>90$ & \\
\hline Sexo & $85(32,7)$ \\
Masculino & $175(67,3)$ \\
Feminino & \\
\hline Raça/Cor & $168(64,6)$ \\
Branco & $38(14,6)$ \\
Preta & $54(20,8)$ \\
Parda & \\
Situação Familiar & $147(56,5)$ \\
Convive com Companheiro (a) e filho (a) & $62(23,8)$ \\
Convive c/ familiares, s/ companheiro & $51(19,6)$ \\
Vive só &
\end{tabular}


Continuação da Tabela 1

\begin{tabular}{ll}
\hline Variáveis & $\mathrm{n}(\%)$ \\
\hline Escolaridade & $25(9,6)$ \\
Não sabe ler/escrever & $163(62,7)$ \\
Ensino Fundamental & $61(23,5)$ \\
Ensino Médio & $11(4,2)$ \\
Ensino Superior & \\
\hline Ocupação & $67(25,8)$ \\
Não aposentado & $193(74,2)$ \\
Aposentado (a)/ Pensionista & \\
\hline ABEP - Associação Brasileira de Empresas de Pesquisa & $95(36,5)$ \\
AB - Alto poder de compra & $106(40,8)$ \\
C - Baixo poder de compra & $59(22,7)$ \\
DE - Poder de compra muito baixo &
\end{tabular}

Das 260 pessoas idosas, $66(25,4 \%)$ faziam uso de psicotrópicos, prevalecendo o consumo de clonazepam $(23,9 \%)$, do grupo dos benzodiazepínicos, e amitriptilina (22,8\%), pertencente a classe dos antidepressivos tricíclicos.
Os inibidores seletivos de recaptação da serotonina foram representados pela prevalência do uso da fluoxetina $(21,7 \%)$ e outros antidepressivos atípicos foram encontrados na análise, como o bupropiona $(2,2 \%)$ (Tabela 2$)$.

Tabela 2. Classe dos psicotrópicos utilizados pelas pessoas idosas com hipertensão acompanhadas pela APS (N=66). Paraná, Brasil, 2016.

\begin{tabular}{lll}
\hline Variáveis & Código ATC & $\mathrm{N}(\%)^{*}$ \\
\hline Benzodiazepínicos & N05BA & $22(33,3)$ \\
Clonazepan & N03AE01 & $8(12,1)$ \\
Bromazepan & N05BA08 & $2(3,1)$ \\
Diazepan & N05BA01 & \\
\hline Antidepressivos & N06A/N06AX/N06AB & $21(31,8)$ \\
Amitriptilina & N06AA09 & $3(4,5)$ \\
Imipramina & N06AA02 & $2(3,1)$ \\
Clomipramina & N06AA04 & $2(3,1)$ \\
Bupropiona & N06AX12 & $1(1,5)$ \\
Mirtizapina & N06AX11 & $1(1,5)$ \\
Venlafaxina & N06AX16 & $20(30,3)$ \\
Fluoxetina & N06AB03 & $5(5,4)$ \\
Paroxetina & N06AB05 & $3(4,5)$ \\
Sertralina & N06AB06 & $2(3,1)$ \\
Citalopram & N06AB04 & \\
\hline
\end{tabular}

ATC: Anatomical Therapentic Chemical Classification System; $*$ Participantes faziam uso de mais um tipo de medicamentos, valor de $\mathrm{n}$ não ponderado. 
Na Tabela 3 observa-se que as pessoas idosas com idade entre 80 e 89 anos possuem menor prevalência no uso de psicotrópicos (RP: 0,32; IC95\%: 0,10-0,99). Ainda no contexto demográfico, a análise apontou que os idosos do sexo feminino (RP: 2,14; IC95\%: 1,11-4,14), que convivem com familiares sem companheiro (RP: 0,57; IC95\%: $0,27-1,21)$ e pertencentes a classe C (RP: 1,85 ; IC 95\%: 0,95-3,57) possuem maior prevalência no uso de psicotrópicos. No que concerne as questões dos hábitos de vida, evidenciou-se maior prevalência no uso dos medicamentos para os que fazem uso de tabaco (PR: 3,12; IC95\%: 1,48-6,57); álcool (RP: 3,03; IC95\%: 0,59-15,4) e que não realizam atividades físicas regularmente (RP: 1,79; IC95\%: $0,91-3,49)$. No contexto clínico, a prevalência significativa no uso psicotrópicos foi maior para as pessoas idosas em uso de polifarmácia (RP: 0,66; IC95\%: 0,36-1,20), que referem efeitos adversos da medicação (RP: 2,03; IC95\%: 0,91-4,49) e para aquelas que foram classificadas com obesidade (OR: 2,05; IC95\%: 1,06-3,97).

Tabela 3. Análise univariada de fatores associados ao consumo de psicotrópicos entre pessoas idosas com hipertensão acompanhados pela APS ( $\mathrm{n}=260)$. Paraná, Brasil, 2016.

\begin{tabular}{|c|c|c|c|c|c|}
\hline \multirow[b]{2}{*}{ Variáveis independentes } & \multicolumn{2}{|c|}{ Uso de Psicotrópicos } & \multicolumn{3}{|c|}{ Análise Univariada } \\
\hline & $\begin{array}{l}\operatorname{Sim}(n=66) \\
n(\%)\end{array}$ & $\begin{array}{l}\text { Não }(\mathrm{n}=194) \\
\mathrm{n}(\%)\end{array}$ & OR & IC95\% & $p$ \\
\hline \multicolumn{6}{|l|}{ Perfil Sociodemográfico e econômico } \\
\hline \multicolumn{6}{|l|}{ Idade (em anos) } \\
\hline $60-69$ & $36(54,5)$ & $91(46,9)$ & 1 & & \\
\hline $70-79$ & $26(36,4)$ & $70(36,1)$ & 0,93 & $00,51-1,69$ & 0,835 \\
\hline $80-89$ & $4(6,1)$ & $31(16)$ & 0,32 & $0,10-0,99$ & $0,048^{*}$ \\
\hline$>90$ & $-(-)$ & $2(1)$ & - & - & - \\
\hline \multicolumn{6}{|l|}{ Sexo } \\
\hline Masculino & $14(21,2)$ & $71(36,6)$ & 1 & & \\
\hline Feminino & $52(78,2)$ & $123(63,4)$ & 2,14 & $1,11-4,14$ & $0,023^{*}$ \\
\hline \multicolumn{6}{|l|}{ Raça/cor } \\
\hline Branco & $40(60,6)$ & $128(66)$ & 1 & & \\
\hline Pardo & $16(24,2)$ & $38(19,6)$ & 1,14 & $0,51-2,55$ & 0,745 \\
\hline Preto & $10(15,2)$ & $28(14,4)$ & 1,34 & $0,68-2,66$ & 0,393 \\
\hline \multicolumn{6}{|l|}{ Escolaridade } \\
\hline Não alfabetizado & $4(6,1)$ & $21(10,8)$ & 1 & & \\
\hline Ensino fundamental & $46(69,7)$ & $117(60,3)$ & 2,06 & $0,67-6,34$ & 0,206 \\
\hline Ensino médio & $14(21,2)$ & $47(24,2)$ & 1,56 & $0,46-5,32$ & 0,474 \\
\hline Ensino superior & $2(3)$ & $9(4,6)$ & 1,16 & $0,18-7,55$ & 0,872 \\
\hline \multicolumn{6}{|l|}{ Situação Familiar } \\
\hline Convive com companheiro e filho & $40(60,6)$ & $107(55,2)$ & 1 & & \\
\hline Convive com familiares sem companheiro & $11(16,7)$ & $51(26,3)$ & 0,57 & $0,27-1,21$ & $0,148^{*}$ \\
\hline Vive só & $15(22,7)$ & $36(18,6)$ & 1,11 & $0,55-2,25$ & 0,762 \\
\hline \multicolumn{6}{|l|}{ Ocupação } \\
\hline Aposentado & $55(83,3)$ & 161(83) & 1 & & \\
\hline Não Aposentado & $11(16,7)$ & $33(17)$ & 1,02 & $0,48-2,16$ & 0,949 \\
\hline \multicolumn{6}{|l|}{$\begin{array}{l}\text { ABEP: Associação brasileira de empresas de } \\
\text { pesquisa }\end{array}$} \\
\hline AB: alto poder de compra & $18(27,3)$ & $77(39,7)$ & 1 & & \\
\hline C: médio poder de compra & $32(48,5)$ & $74(38,1)$ & 1,85 & $0,95-3,57$ & $0,068^{*}$ \\
\hline DE: baixo poder de compra & $16(24,2)$ & $43(22,2)$ & 1,59 & $0,73-3,43$ & 0,237 \\
\hline
\end{tabular}


Continuação da Tabela 3

\begin{tabular}{|c|c|c|c|c|c|}
\hline \multirow[b]{2}{*}{ Variáveis independentes } & \multicolumn{2}{|c|}{ Uso de Psicotrópicos } & \multicolumn{3}{|c|}{ Análise Univariada } \\
\hline & $\begin{array}{l}\operatorname{Sim}(n=66) \\
n(\%)\end{array}$ & $\begin{array}{l}\text { Não (n=194) } \\
\mathrm{n}(\%)\end{array}$ & OR & IC95\% & $p$ \\
\hline \multicolumn{6}{|c|}{ Perfil clínico, medicamentoso e bábitos de vida } \\
\hline \multicolumn{6}{|c|}{ Controle Pressórico } \\
\hline Adequado & $34(51,5)$ & $95(49)$ & 1 & & \\
\hline Inadequado & $32(48,5)$ & $99(51)$ & 0,9 & $0,51-1,57$ & 0,721 \\
\hline \multicolumn{6}{|c|}{ Sequelas decorrentes da HA } \\
\hline Não & $54(81,8)$ & $171(88,1)$ & 1 & & \\
\hline Sim & $12(18,2)$ & $23(11,9)$ & 1,65 & $0,77-3,54$ & $0,197 *$ \\
\hline \multicolumn{6}{|l|}{ Tabagista } \\
\hline Não & $50(75,8)$ & $176(90,7)$ & 1 & & \\
\hline $\operatorname{Sim}$ & $16(24,2)$ & $18(9,3)$ & 3,12 & $1,48-6,57$ & $0,003^{*}$ \\
\hline \multicolumn{6}{|l|}{ Etilismo } \\
\hline Não & $63(95,5)$ & $191(98,5)$ & 1 & & \\
\hline $\operatorname{Sim}$ & $3(4,5)$ & $3(1,5)$ & 3,03 & $0,59-15,4$ & $0,181 *$ \\
\hline \multicolumn{6}{|l|}{ Prática de atividade física } \\
\hline Ativo & $16(24,2)$ & $58(29,9)$ & 1 & & \\
\hline Sedentário & $41(62,1)$ & $83(42,8)$ & 1,79 & $0,91-3,49$ & $0,087^{*}$ \\
\hline Moderadamente ativo & $9(13,6)$ & $53(27,3)$ & 0,61 & $0,25-1,51$ & 0,289 \\
\hline \multicolumn{6}{|l|}{ Polifarmácia } \\
\hline Não & $45(68,2)$ & $114(58,8)$ & 1 & & \\
\hline $\operatorname{Sim}$ & $21(31,8)$ & $80(41,2)$ & 0,66 & $0,36-1,20$ & $0,177 *$ \\
\hline \multicolumn{6}{|c|}{ Efeitos adversos da medicação hipertensiva } \\
\hline Não & $46(69,7)$ & $148(76,3)$ & 1 & & \\
\hline Sim & $12(18,2)$ & $19(9,8)$ & 2,03 & $0,91-4,49$ & $0,080^{*}$ \\
\hline Às vezes & $8(12,1)$ & $27(13,9)$ & 0,95 & $0,40-2,24$ & 0,913 \\
\hline \multicolumn{6}{|l|}{ Antropometria } \\
\hline \multicolumn{6}{|c|}{ Classificação do Índice de Massa Corporal } \\
\hline Normal & $16(24,2)$ & $46(23,7)$ & 1 & & \\
\hline Excesso de peso & $31(47)$ & $65(33,5)$ & 1,37 & $0,67-2,79$ & 0,385 \\
\hline Obesidade & $19(28,8)$ & $83(42,8)$ & 2,05 & $1,06-3,97$ & $0,031^{*}$ \\
\hline \multicolumn{6}{|l|}{ Circunferência Abdominal } \\
\hline Normal & $15(22,7)$ & $74(38,1)$ & 1 & & \\
\hline Alterada & $51(77,3)$ & $120(61,9)$ & 2,09 & $1,10-3,99$ & $0,024^{*}$ \\
\hline \multicolumn{6}{|l|}{ Razão cintura-estatura } \\
\hline Normal & $9(13,6)$ & $32(16,5)$ & 1 & & \\
\hline Alterada & $55(83,4)$ & $162(83,5)$ & 1,2 & $0,54-2,68$ & 0,645 \\
\hline \multicolumn{6}{|l|}{ Índice de Conicidade } \\
\hline Normal & $6(9,1)$ & $13(6,7)$ & 1 & & \\
\hline Alterada & $60(90,9)$ & $181(93,3)$ & 0,71 & $0,26-1,97$ & 0,521 \\
\hline
\end{tabular}

OR: Odds Ratio; IC: índice de confiança; *Variáveis que apresentaram valor de $\mathrm{p}<0,20$ foram inseridas na etapa multivariada, conforme o modelo Stepwise de regressão logística. 
Quanto ao perfil clínico e antropométrico, a análise multivariada evidenciou que as pessoas idosas que autorreferiram realizar uso diário de tabaco (RP: 4,09; IC95\%: 1,81-9,18), apresentaram CA alterada (RP: 2,58; IC95\%: 1,29-5,18), classificadas com obesidade (RP: 2,43; IC95\%: 1,30-4,55) e que declararam ter efeitos colaterais dos medicamentos utilizadas no tratamento da HA (OR: 2,98; IC95\%: 1,23-7,21), possuem maior prevalência de fazerem uso de psicotrópicos (Tabela 4). No seguimento das questões organizacionais e relacionais das equipes da APS, observa-se que as pessoas idosas insatisfeitas com o atendimento (RP: 6,71; IC95\%: 1,37-32,71) que não se sentiam apoiadas e que compreendidas pelos profissionais de saúde aos problemas durante as consultas (RP: 2,17; IC95\%: 1,11-4,25), possuem maior prevalência de fazer uso de psicotrópicos, ajustado pela variável ABEP (Tabela 4).

Tabela 4. Análise multivariada de fatores associados ao consumo de psicotrópicos entre pessoas idosas com hipertensão acompanhados pela APS (N = 260). Paraná, Brasil, 2016.

\begin{tabular}{|c|c|c|c|c|c|}
\hline \multirow[b]{2}{*}{ Variáveis } & \multicolumn{2}{|c|}{ Uso de Psicotrópicos } & \multicolumn{3}{|c|}{ Análise Multivariada } \\
\hline & $\begin{array}{l}\operatorname{Sim}(\mathrm{n}=66) \\
\mathrm{n}(\%)\end{array}$ & $\begin{array}{l}\text { Não (n=194) } \\
\mathrm{n}(\%)\end{array}$ & OR & IC95\% & $p$ \\
\hline \multicolumn{6}{|l|}{ Tabagista } \\
\hline Não & $50(75,8)$ & $176(90,7)$ & 1 & & \\
\hline Sim & $16(24,2)$ & $18(9,3)$ & 4,09 & $1,81-9,18$ & 0,001 \\
\hline \multicolumn{6}{|l|}{ Circunferência Abdominal } \\
\hline Normal & $15(22,7)$ & $74(38,1)$ & 1 & & \\
\hline Alterada & $51(77,3)$ & $76(61,9)$ & 2,58 & $1,29-5,18$ & 0,005 \\
\hline \multicolumn{6}{|l|}{ Obesidade } \\
\hline Não & $28(42,4)$ & $118(60,8)$ & 1 & & \\
\hline Sim & $38(57,6)$ & $76(39,2)$ & 2,43 & $1,30-4,55$ & 0,007 \\
\hline \multicolumn{6}{|c|}{ Efeitos adversos da medicação hipertensiva } \\
\hline Não & $46(69,7)$ & $148(76,3)$ & 1 & & \\
\hline $\operatorname{Sim}$ & $12(18,2)$ & $19(9,8)$ & 2,98 & $1,23-7,21$ & 0,015 \\
\hline As vezes & $8(12,1)$ & $27(13,9)$ & 1,29 & $0,51-3,27$ & 0,589 \\
\hline \multicolumn{6}{|c|}{$\begin{array}{l}\text { ABEP: Associação brasileira de empresas } \\
\text { de pesquisa }\end{array}$} \\
\hline AB: alto poder de compra; & $18(27,3)$ & $77(39,7)$ & 1 & & \\
\hline C: médio poder de compra & $32(48,5)$ & $74(38,1)$ & 1,94 & $0,93-4,05$ & 0,074 \\
\hline DE: baixo poder de compra. & $16(24,2)$ & $43(22,2)$ & 1,71 & $0,73-4,00$ & 0,212 \\
\hline
\end{tabular}

OR: Odds Ratio; IC: índice de confiança; *Variáveis explicativas ajustada pela variável ABEP.

\section{DISCUSSÃO}

Sabe-se que atualmente, com o avanço na expectativa de vida da população, a demanda das pessoas idosas na busca por serviços de saúde em geral, principalmente na APS, também aumentou. A predominância do sexo feminino neste estudo, pode ser justificada pela maior preocupação das mulheres com a saúde, maior facilidade para descrever os problemas físicos e psicológicos, fato este que eleva a probabilidade de receber e seguir prescrições médicas. Além de ser considerado o sexo mais afetado por problema de saúde não fatal, e que convive por maior tempo com doenças crônicas ${ }^{20,21}$.

Em Campinas-São Paulo, estudo realizado na atenção primária apontou que os psicotrópicos também foram mais utilizados por mulheres, indivíduos de raça/cor branca, com pior percepção da saúde, transtornos mentais comuns e problemas 
emocionais ${ }^{6}$. Em estudo realizado em Curitiba, capital do Paraná, com mulheres idosas e que vivem com companheiro, tem baixo nível de escolaridade e sem ocupação formal (donas de casa) possuem maior prevalência no uso de psicotrópicos ${ }^{22}$.

No que se refere a escolaridade dos participantes do estudo, esse resultado condiz com achados que atribuem o nível de escolaridade ao conhecimento sobre saúde e serviços de saúde ${ }^{20-22}$. Desta forma, as pessoas idosas com baixa escolaridade possuem mais dificuldades no autocuidado e na autopercepção das necessidades de saúde, reduzindo a autonomia na busca por cuidados e tratamento médico ${ }^{20-22}$.

Em relação as questões econômicas, a maioria das pessoas idosas pertencia ao extrato $\mathrm{C}$, o que significa baixo poder de compra do chefe familiar. $\mathrm{O}$ adoecimento psiquico ou a utilização predominante de psicotrópicos pelas classes economicamente mais baixas se justificam na literatura diante a vulnerabilidade social, a partir do acesso limitado a serviços de saúde, lazer, alimentação adequada, baixo rendimento econômico, saneamento basico, educação e prática de exercicios fisicos, que favorecem o surgimento de doenças crônicas, agravamentos, e hospitalizações s,20,21 $^{9}$.

Estudo realizado com idosos que utilizam psicofármacos prescritos por profissionais do SUS e por profissionais de plano de saúde suplementar (PSS), verificou que os mais utilizados pelas pessoas idosas usuárias do Sistema Único de Saúde (SUS) são aqueles de menor custo e que na maioria das vezes causam mais efeitos colaterais, enquanto os prescritos no PSS são medicamentos com menor risco de efeitos indesejáveis. Apesar de não existirem diferenças significativas entre a quantidade de psicofármacos utilizada pelos usuários de ambos os sistemas, há a sinalização da falta de acesso dos usuários do SUS aos medicamentos mais modernos e que proporcionem menos efeitos adversos ${ }^{23}$.

No que tange aos psicotrópicos mais utilizados pelos participantes deste estudo, foi verificado o uso de benzodiazepínicos, antidepressivos tricíclicos, inibidores seletivos de recaptação da serotonina e outros antidepressivos. Esses dados podem ser corroborados por pesquisas que destacam a prevalência do uso da fluoxetina ${ }^{6}$, clonazepam, amitriptilina, fluoxetina e bupropiona pela população idosa ${ }^{3,4,24}$. Importante frisar que o uso da bupropiona neste estudo, pode estar relacionado como apoio medicamentoso no tratamento ao tabagismo constantes no Protocolo Clínico e Diretrizes Terapêuticas - Dependência à Nicotina, já que é um medicamento considerado como $1^{a}$ linha no tratamento da dependência à nicotina utilizado no Brasil ${ }^{25}$.

Neste sentido, os hábitos de prescrição para os usuários do SUS costumam ser influenciados pela relação de medicamentos disponíveis gratuitamente ${ }^{24,25}$. Estudo realizado em município do Oeste Paulista, aponta que os usuários do SUS utilizam, em maior proporção, os mesmos medicamentos descritos neste estudo ${ }^{26}$, e que também estão presentes na Relação Nacional de Medicamentos Essenciais (RENAME), base de aquisição de medicamentos fornecidos pelo SUS e distribuídos pelas equipes da APS $^{27}$.

Ressalta-se que nesta pesquisa, os resultados sobre o uso de polifarmácia foram diagnosticados em $25 \%$ da população estudada. Tal fato, pode ser corroborado em estudo com idosos na APS em Belo Horizonte, justificado pelo envelhecimento progressivo e a tendência de aumento ao uso de medicamentos. Logo, o desenvolvimento de ações no sentido de garantir uma farmacoterapia adequada para a pessoa idosa, devem ser prioridade. Medidas combinadas abrangendo estratégias de revisão da farmacoterapia, sistema informatizado de apoio a prescrição e dispensação, educação continuada para a equipe de saúde e serviços especializados em geriatria são importantes para garantir uma prescrição adequada $^{28}$.

Nessa perspectiva, os profissionais da APS tem como objetivos realizar ações de prevenção, promoção e educação em saúde, incluindo ações direcionadas aos usuários que fazem uso de medicamentos psicotrópicos. Atividades de orientação em saúde devem ser priorizadas, principalmente em relação aos possíveis efeitos adversos que podem ocorrer no uso conjunto de psicofármacos com outras classes medicamentosas, enfatizando a prevenção dos abusos e a promoção do uso racional ${ }^{13,23}$. 
Alguns pesquisadores relatam que apesar da prevalência do uso de psicotrópicos na população senil ser alta, algumas pessoas respondem as monoterapias farmacológicas com drogas mais leves e menos prejudiciais à saúde que podem ser atreladas a terapias não-farmacológicas, em especial na APS, por intermédio das equipes da ESF. No entanto, fazse necessário ter cautela na abordagem, no tocante as interações medicamentosas, devido ao fato de as pessoas idosas serem suscetíveis a polifarmácia, expondo-as aos riscos de quedas e fraturas devido ao grau de sedação, bem como perda da capacidade cognitiva e da memória ${ }^{3-5}$.

$\mathrm{Na}$ análise multivariada deste estudo foi encontrada associação entre o consumo de psicotrópicos e ser tabagista, ter excesso de peso, CA alterada, obesidade e feitos adversos ao medicamento anti-hipertensivo. Estudo que analisou a qualidade de vida em pessoas idosas atendidas pela APS no Acre, detectou associação sobre a menor percepção da qualidade de vida com diagnóstico clínico de multimorbidades e depressão. Assim, torna-se um desafio para os profissionais da APS desenvolverem cuidado eficaz de modo a reduzir os impactos da senilidade na qualidade de vida ${ }^{29}$.

E se referindo aos tratamentos disponíveis para excesso de peso encontramos medicamentos das classes de ansioliticos e antidepressivos. Uma das práticas centrais para o manejo de doenças crônicas na APS, tem como objetivo o controle da obesidade, que detém entre as principais recomendações, o incentivo à mudança de estilo de vida, começando com a prática de atividades físicas, reeducação alimentar, abandono do tabaco entre outras ${ }^{30}$. Além disso, evidencia científica associação bidirecional entre excesso de peso e diversos disturbios psiquiátricos, cuja chances de um individuo com excesso de peso desenvolver depressão, é semelhante ao de um sujeito com depressão tornar-se obeso ${ }^{31}$.

Deste modo, o acompanhamento da pessoa idosa deve levar em conta a natureza multifatorial e a complexidade dos agravos nessa fase da vida, considerando as diferenças nas percepções dessa população em relação ao excesso de peso, destacando as fragilidades emocionais diante a multimorbidade, mudanças de percepção sobre envelhecimento e saúde, e valorização da funcionalidade ${ }^{30,31,32}$.
O tabagismo também é um fator que pode estar associado a um maior risco de desenvolver distúrbios mentais, entre eles a depressão. $\mathrm{Na}$ literatura, a causalidade entre tabagismo e depressão é atribuída a mecanismos distintos, destacando à ação da nicotina nos sistemas neuroquímicos, bem como nas funções neuroendócrinas. Também se tem uma causalidade inversa, em que indivíduos com depressão podem iniciar e manter o uso de derivados do tabaco, e isto pode ser explicado pela ausência de comportamentos para autopreservação do estado de saúde e/ou pelo aumento das sensações prazerosas ao fumar ${ }^{33}$.

A realização de atividades de educação em saúde direcionadas as pessoas idosas, com a participação da família, torna-se uma ferramenta indispensável aos profissionais de saúde, para que os idosos compreendam o agravamento que o uso crônico do fumo e álcool podem trazer. Destarte, essa faixa etária faz parte de um grupo onde é recorrente os problemas de saúde advindos com a idade, aumentando a susceptibilidade as doenças crônicas. Quando associado ao uso da nicotina e do álcool, essas pessoas ficam mais propensas às internações e agravamento de um quadro patológico ${ }^{34}$.

Ademais, as questões relacionais entre profissional de saúde e usuário podem influenciar o modo de utilização dos psicofármacos, considerando que o vínculo entre eles se torna essencial para a monitorização do paciente. Estudo realizado com idosos octogenários que avaliaram os serviços de cuidados primários, relataram aspectos de insatisfação relacionados à demora no atendimento e à infraestrutura da unidade. Porém ficaram satisfeitos com o atendimento dos Agentes Comunitários de Saúde, por serem atenciosos, explicarem o que deve ser feito, interessarem-se pelos seus problemas e estarem disponíveis ${ }^{35}$.

Os benefícios das orientações profissionais destinadas aos pacientes com hipertensão arterial, podem ser constatados por estudo que fez com que os níveis da pressão arterial dos usuários diminuíssem durante o manejo do autocuidado. Na questão do uso de psicotrópicos, mesmo não havendo significância estatística com o controle pressórico inadequado, é importante destacar que a metade dos entrevistados com a hipertensão são usuários consumidores de psicotrópicos ${ }^{4}$. 
Estudo realizado no sul do Brasil constatou que a falta de adesão e vínculo precário com a equipe de APS teve forte associação com o controle pressórico inadequado e a ausência nas consultas de rotina realizadas pelos programas de hipertensão. Não obstante, a evidência aponta a necessidade de reorganização das ações e intervenções em saúde, pautada na necessidade de os profissionais realizarem atendimento de maneira integral e interdisciplinar e não apenas pautadas nas questões sintomáticas e/ ou complicações oriundas do descontrole pressórico, para que haja maior envolvimento dos idosos com hipertensão nas consultas de rotina e adesão à terapêutica medicamentosa ${ }^{13}$.

Considera-se como limitação do estudo sua abordagem metodológica, devido ao viés de casualidade reversa, que pode ter omitido alguns dados importantes para a elucidação da temática desta pesquisa. No entanto, o estudo avança nas questões relacionadas ao atendimento prestado a pessoa idosa, estimulando os profissionais de saúde a conhecerem os fatores associados ao uso de psicotrópicos pela pessoa idosa com comorbidades, a fim de qualificar $\mathrm{o}$ atendimento para essa população.

\section{CONCLUSÃO}

Os resultados do estudo apontaram que 25\% da população estudada faz uso de psicotrópicos, sendo as classes dos antidepressivos e ansiolíticos os mais predominantes. Os medicamentos mais utilizados foram do grupo dos benzodiazepínicos, antidepressivos tricíclicos, inibidores da recaptação

\section{REFERÊNCIAS}

1. Naloto DCC, Lopes FC, Barberato Filho S, Lopes LC, Fiol FSD, Bergamaschi CC. Prescription of benzodiazepines for adults and the elderly of a mental health outpatient clinic. Ciênc Saúde Colet. 2016;21(4:1-10). Disponível em: https://doi. org/10.1590/1413-81232015214.10292015.

2. Alvim MM, Cruz DT, Vieira MT, Bastos RR, Leite ICG. Prevalence and factors associated with the use of benzodiazepines in the community elderly. Rev Bras Geriat. Gerontol. 2017;20(4):463-74. Disponível em: https://doi.org/10.1590/1981-22562017020.170042 . da serotonina e outros antidepressivos. Os fatores associados ao consumo de psicotrópicos entre pessoas idosas com hipertensão arterial acompanhados pela atenção primaria foram o uso diário de tabaco, sedentário, obesidade, circunferência abdominal alterada e autorrelato de efeitos adversos ao medicamento anti-hipertensivo.

Por ser um problema de saúde pública que atinge parcela significativa da população com maior risco de agravos a saúde, destaca-se a importância de pesquisas nessa área, para que busquem alternativas para melhorar a qualidade de vida da pessoa idosa com comorbidades e que utilizam psicotrópicos, por meio de um cuidado integral e equânime, o qual reduza as iniquidades assistenciais à população idosa que convive com doenças crônicas.

Os hábitos de vida não saudáveis observados como fatores de maior prevalência no uso de psicotrópicos são modificáveis e podem ser trabalhados com diferentes práticas intervencionistas, elucidadas pelos profissionais de saúde que integram as equipes multiprofissionais presentes nas unidades de atenção primária. A organização do processo de trabalho baseia-se no conhecimento populacional e nas ações de cunho educacional e orientativas, apontando a importância da adoção de novos hábitos alimentares e de atividade física, que podem reduzir a polifarmácia. Desta forma, os resultados apresentados nesta pesquisa podem reforçar o direcionamento assistencial com maior qualidade e resolutivos aos problemas de saúde identificados na população.

Editado por: Ana Carolina Lima Cavaletti
3. Borges TL, Vedana KGG, Castilho ECD, Miasso AI. Factors associated with potential drug-drug interactions in patients attended in Primary Health Care: a focus on mental health. Issues Mental Health Nurs. 2017;38:769-74. Disponível em: https://doi.org /10.1080/01612840.2017.1350771.

4. Silva PA, Almeida LY, Souza J. The use of benzodiazepines by women cared for at a Family Health Unit. Rev Esc Enferm USP. 2019;53:e03419. Disponível em: https://doi.org/10.1590/s1980$220 \times 2017038903419$. 
5. Maree RD, Marcum ZA, Saghafi E, Weiner DK, Karp JF. A systematic review of opioid and benzodiazepine misuse in older adults. Am J Geriatr Psychiatry 2016;24:949-63. Disponível em: https:// doi.org/10.1016/j.jagp.2016.06.003 .

6. Prado MAMB, Francisco PMSB, Barros MBA. Use of psychotropic medications in adults and elderly living in Campinas, São Paulo, Brazil: cross-sectional population-based study. Epidemiol Serv Saúde. 2017;26(4):747-58. Disponível em: http://dx.doi. org/10.5123/s1679-49742017000400007 .

7. Mann E, Köpke S, Haastert B, Pitkälä K, Meyer G. Psychotropic medication use among nursing home residents in Austria: a cross-sectional study. BMC Geriatrics. 2009;9:1-10. Disponível em: https://doi. org/10.1186/1471-2318-9-18.

8. Álvares LM, Lima RC, Silva RA. Occurrence of falls in elderly residents in long-term care institutions in Pelotas Rio Grande do Sul. Cad Saúde Pública. 2010;26(1):31-40. Disponível em: https://doi. org/10.1590/S0102-311X2010000100004 .

9. Abi-Ackel MM, Lima-Costa MF, Castro-Costa E, Loyola Filho AI. Use of psychotropic drugs among elderly residents in the community: prevalence and associated factors. Rev Bras Epidemiol. 2017;20(1):110. Disponível em: https://doi.org/10.1590/19805497201700010005 .

10. Paraná. Instituto Paranaense de Desenvolvimento Econômico e Social -IPARDES. Caderno Estatístico Município de Maringá [Internet]. Curitiba: IPARDES; 2016 [acesso 15 mar. 2020]. Disponível em: http:// www.ipardes.gov.br/cadernos/MontaCadPdf1. php? Municipio $=87000 \& \mathrm{btOk}={ }_{\mathrm{ok}}$.

11. Paes NA, Silva CS, Figueiredo TMRM, Cardoso MAA, Lima JO. Satisfaction of hypertensive users with primary care network services in Brazil: a validation study. Rev Panam Salud Publica. 2014;36(2). Disponível em: https://www.scielosp. org/article/rpsp/2014.v36n2/87-93 .

12. Associação Brasileira de Empresas de Pesquisa. Adoção do CCEB 2008: Critério de Classificação Econômica Brasil. São Paulo: ABEP; 2015 [acesso 09 mar. 2020]. Disponível em: file:///C:/Users/pse/ Downloads/05_cceb_2008_em_vigor_em_2010_ base_1se_2008.pdf.

13. Rêgo AS, Radovanovic CAT. Adherence of hypertension patients in the Brazil's Family Health Strategy. Rev Bras Enferm. 2018;71:1030-7. Disponível em: https://doi.org/10.1590/0034-71672017-0297 .
14. World Health Organization Collaborating Centre for Drugs Statistics Methodology. Anatomical Therapeutic Chemical Classification: ATC Code [Internet]. Oslo: WHO; 2016 [acesso 15 mar. 2020]. Disponível em: http://www.whocc. no/ atc_ddd_index/.

15. Malachias MVB, Souza WKSB, Plavnik FL, Rodrigues CIS, Brandão AA, Neves MFT, et al. Sociedade Brasileira de Cardiologia. $7^{\mathrm{a}}$ Diretriz Brasileira de Hipertensão Arterial. Arq Bras Cardiol. 2016];107(3):1-104. Disponível em: http://www. scielo.br/pdf/abc/v107n3s3/0066-782X-abc-10703-s3-0067.pdf .

16. Associação Brasileira para o Estudo da Obesidade e da Síndrome Metabólica. Diretrizes brasileiras de obesidade 2016 [Internet]. $4^{a}$ ed. São Paulo; 2016 [acesso 16 set. 2016]. Disponível em: http://www.abeso.org.br/uploads/ downloads/92/57fccc403e5da.pdf .

17. Brasil. Ministério da Saúde, Secretaria de Atenção à Saúde, Departamento de Ações Programáticas e Estratégicas. Caderneta de Saúde da Pessoa Idosa: manual de preenchimento [Internet]. Brasília, DF: MS; 2014 [acesso em 03 ago. 2016]. Disponível em: http://189.28.128.100/dab/docs/publicacoes/ geral/ pnab.pdf .

18. Corrêa MM, Tomasi E, Thumé E, Oliveira ERA, Facchini LA. Waist-height ratio as an anthropometric marker of overweight in Elderly Brazilians. Cad Saúde Pública 2017;33:1-10. Disponível em: https://doi. org/10.1590/0102-311x00195315.

19. Pitanga FJG, Lessa I. Sensitivity and specificity of the conicity index as a coronary risk predictor among adults in Salvador, Brazil. Rev Bras Epidemiol. 2004;7:259-69. Disponível em: https://doi. org/10.1590/S1415-790X2004000300004.

20. Araujo BG, Moraes CF, da Fonseca KA. of the use of potentially inappropriate drugs for the elderly in Brazil: a systematic review. Rev Kairós. 2019;22:11939. Disponível em: https://doi.org/10.23925/2176901X.2019v22i4p119-139 .

21. Fernandes CSE, Azevedo RCS, Goldbaum M, Barros MBA. Psychotropic use patterns: Are there differences between men and women? PLoS ONE 2018;13:e0207921. Disponível em: https://doi. org/10.1371/journal.pone.0207921 .

22. Claro MP, Tashima CM, Dalcól C, Katakura EALB. Psychotropic prescription profile in a basic health unit in Paraná. Braz J Develop. 2020;6(7):44451-65. Disponível em: https://doi.org/10.34117/bjdv6n7-167. 
23. Almeida GMF, Lazarini CA, Stefano ICA, Muniz ECS, Panes VCB, Marin MJS. Uso de psicofármacos entre idosos usuários do sistema único de saúde e do plano de saúde suplementar. Estud Interdiscipl Envelhec. 2019;24(2):111-28. Disponível em: https:// seer.ufrgs.br/RevEnvelhecer/article/view/77276.

24. Medeiros Filho JSA, Azevedo DM, Pinto TR, Silva GWS. The use of psychotropic drugs in primary health care. Rev Bras Promoç Saúde, 2018;31(3):1-12. Disponível em: https://periodicos.unifor.br/RBPS/ article/view/7670

25. Brasil. Comissão Nacional de Incorporação de Tecnologias no SUS - CONITEC. Relatório de recomendação: Protocolo Clínico e Diretrizes Terapêuticas do Tabagismo [Internet]. Brasília, DF: 2020 [acesso 09 maio 2020]. Disponível em: http:// conitec.gov.br/images/Relatorios/2020/Relatrio_ PCDT_Tabagismo_520_2020_FINAL.pdf

26. Brasil. Ministério da Saúde. Secretaria de Vigilância em Saúde, Departamento de Análise em Saúde e Vigilância de Doenças Não Transmissíveis. Vigitel Brasil 2019: vigilância de fatores de risco e proteção para doenças crônicas por inquérito telefônico: estimativas sobre frequência e distribuição sociodemográfica de fatores de risco e proteção para doenças crônicas nas capitais dos 26 estados brasileiros e no Distrito Federal em 2019. Brasília, DF: MS; 2020.

27. Oliveira PC, Silveira MR, Ceccato MGB, Reis AMM, Pinto IVL, Reis EA. Prevalence and Factors Associated with Polypharmacy in Elderly People attended in Primary Health Care in Belo HorizonteMG, Brasil. Ciênc Saúde Colet. 2021;26(4):1-10. Disponível em: https://doi.org/10.1590/141381232021264.08472019.

28. Abayneh S, Lempp H, Hanlon C. Participatory action Research topilot a model of mental health service user involvement in na Ethiopian rural primary health care setting: study protocol. Res Involv Engagem. 2020;6:1-10. Disponível em: https://doi.org/10.1186/ s40900-019-0175-x.
29. Amaral TLM, Amaral CA, Lima NS, Herculano PV, Prado PR, Monteiro GTR. Multimorbidity, depression and quality of life in elderly people assisted by the Family Health Strategy in Senador Guiomard, Acre, Brazil. Ciênc Saúde Colet. 2018;23(9):1-10. Disponível em: https://doi.org/10.1590/141381232018239.22532016.

30. Malta DC, Bernal RTI, Andrade SSCA, da Silva MMA, Velasquez-Melendez G. Prevalence of and factors associated with self-reported high blood pressure in Brazilian adults. Rev Saúde Pública. 2017;51:1-10. Disponível em: https://doi.org/10.1590/ s1518-8787.2017051000006 .

31. Estrela DC, Mendes BO. Association between obesity and chronic stress: a review on behavioral, biochemical and hematological aspects. Multi-Sci J. 2017;1(9): 41-50. Available from: http://dx.doi.org/10.33837/msj.v1i9.609

32. Machado RET, Braga VAS, Machado DR, Jesus MCP, Oliveira DM, Merighi MAB. Experiences and expectations of obese older people on the care received in the primary health care network. Rev Bras Enferm. 2020;73(Suppl 3):e20200438. Disponível em: http://dx.doi.org/10.1590/0034-7167-2020-0438 .

33. Amorim TA, Lucchese R, Silva Neta EM, Santos JS, Vera I, Paula NI, et al. Determinants of mental health and abuse of psychoactive substances associated with smoking: case study. Ciênc Saúde Coletiva 2019;24:4141-52. Disponível em: https://doi. org/10.1590/1413-812320182411.02752018 .

34. Alexandrino A, Oliveira CBS, Gomes GER, Silva MPGPC, Nogueira MF. Smoking and alcoholism in old age: evaluation of behavioral factors among the elderly. Braz. J Health Rev. 2020;3(2):3275-85. Disponível em: https://doi.org/10.34119/bjhrv3n2-167.

35. Brunström M, Ng N, Dahlström J, Lindholm LH, Lönnberg G, Norberg M, et al. Association of Physician Education and Feedback on Hypertension Management With Patient Blood Pressure and Hypertension Control. JAMA Netw Open. 2020;3:e1918625. Disponível em: https://doi. org/10.1001/jamanetworkopen.2019.18625 . 